\title{
REVISED dbVar structural variant cluster set for data analysis
}

\section{and variant comparison [version 2; peer review: 2 approved]}

\author{
Lon Phan'1, Jeffrey Hsu², Le Quang Minh Tri³ ${ }^{3}$ Michaela Willi4,5, Tamer Mansour6,7, \\ Yan Kai8,9, John Garner ${ }^{1}$, John Lopez ${ }^{1}$, Ben Busby (iD1
}

\footnotetext{
${ }^{1}$ National Center for Biotechnology Information, National Library of Medicine, National Institutes of Health, Bethesda, MD, USA

${ }^{2}$ Cleveland Clinic Lerner Research Institute, Cleveland, OH, USA

${ }^{3}$ Department of Biotechnology, Ho Chi Minh City International University, Ho Chi Minh, Vietnam

${ }^{4}$ Laboratory of Genetics and Physiology, National Institute of Diabetes, Digestive and Kidney Diseases, National Institutes of Health, Bethesda, MA, USA

${ }^{5}$ Division of Bioinformatics, Biocenter, Medical University Innsbruck, Innsbruck, Austria

${ }^{6}$ Lab for Data Intensive Biology, Department of Population Health and Reproduction, University of California, Davis, CA, USA

${ }^{7}$ Department of Clinical Pathology, University of Mansoura, Mansoura, Egypt

${ }^{8}$ Cancer Epigenetics Laboratory, Department of Anatomy and Regenerative Biology, The George Washington University, Washington, DC, USA

${ }^{9}$ Department of Physics, The George Washington University, Washington, DC, USA
}

\section{V2 First published: 13 Apr 2016, 5:673 \\ https://doi.org/10.12688/f1000research.8290.1 \\ Latest published: 28 Feb 2017, 5:673 \\ https://doi.org/10.12688/f1000research.8290.2}

\section{Abstract}

dbVar houses over 3 million submitted structural variants (SSV) from

120 human studies including copy number variations (CNV),

insertions, deletions, inversions, translocations, and complex

chromosomal rearrangements. Users can submit multiple SSVs to

dbVAR that are presumably identical, but were ascertained by different platforms and samples, to calculate whether the variant is rare or common in the population and allow for cross validation.

However, because SSV genomic location reporting can vary including fuzzy locations where the start and/or end points are not precisely known - analysis, comparison, annotation, and reporting of SSVs across studies can be difficult. This project was initiated by the Structural Variant Comparison Group for the purpose of generating a non-redundant set of genomic regions defined by counts of concordance for all human SSVs placed on RefSeq assembly GRCh38 (RefSeq accession GCF_000001405.26). We intend that the availability of these regions, called structural variant clusters (SVCs), will facilitate the analysis, annotation, and exchange of SV data and allow for simplified display in genomic sequence viewers for improved variant interpretation. Sets of SVCs were generated by variant type for each of the 120 studies as well as for a combined set across all studies. Starting from 3.64 million SSVs, 2.5 million and 3.4 million non-redundant SVCs with count $>=1$ were generated by variant type for each study and across all studies, respectively. In addition, we have developed utilities for annotating, searching, and filtering SVC data in

\section{Open Peer Review}

Approval Status

1 2

version 2

(revision)

28 Feb 2017

version 1

13 Apr 2016

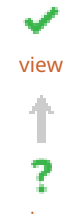

1. Lihua Julie Zhu ID, University of

Massachusetts Medical School, Worcester,

USA

2. Justin M. Zook (iD), National Institute of

Standards and Technology, Gaithersburg,

USA

Any reports and responses or comments on the article can be found at the end of the article. 
GVF format for computing summary statistics, exporting data for genomic viewers, and annotating the SVC using external data sources.

\section{Keywords}

NCBI , dbVar , Structural Variation Cluster , GVF , Genomics, Open-

Source, Genome Annotation, Education, Software

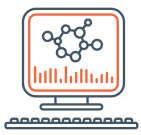

This article is included in the Bioinformatics

gateway.

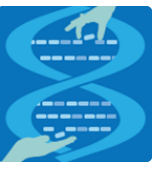

This article is included in the Hackathons

collection.

Corresponding author: Ben Busby (ben.busby@nih.gov)

Competing interests: No competing interests were disclosed.

Grant information: Lon Phan, John Garner, John Lopez, and Ben Busby's work on this project was supported by the Intramural Research Program of the National Institutes of Health (NIH)/ National Library of Medicine (NLM)/NCBI.

The funders had no role in study design, data collection and analysis, decision to publish, or preparation of the manuscript.

Copyright: (c) 2017 Phan L et al. This is an open access article distributed under the terms of the Creative Commons Attribution License, which permits unrestricted use, distribution, and reproduction in any medium, provided the original work is properly cited. The author(s) is/are employees of the US Government and therefore domestic copyright protection in USA does not apply to this work. The work may be protected under the copyright laws of other jurisdictions when used in those jurisdictions.

How to cite this article: Phan L, Hsu J, Tri LQM et al. dbVar structural variant cluster set for data analysis and variant comparison [version 2; peer review: 2 approved] F1000Research 2017, 5:673 https://doi.org/10.12688/f1000research.8290.2

First published: 13 Apr 2016, 5:673 https://doi.org/10.12688/f1000research.8290.1 


\section{REVISED Amendments from Version 1}

The changes to the text are as follows:

1) We addressed referee \#1's question regarding the dbVar update cycle

2) We address referee \#1's questions about the percent total reported

3) We added additional references for the manuscript

4) We added clearer images for the figures

We would like to thank the reviewers for taking the time to review this paper!

See referee reports

\section{Introduction}

There is a growing body of evidence suggesting that genomic structural variants play an important role in the etiology of human disease and in determining individuals' characteristics and phenotypes ${ }^{1,2}$. Structural variants are also important for understanding the evolution of species ${ }^{3}$. dbVar is a database of large structural genomic variants that catalogs millions of records from both small and large studies and makes them freely available to the public ${ }^{4,5}$. The data are organized by submitted study, which makes for convenient comparisons between cases and controls. dbVar online search and browser tools make it easy to search and retrieve the data.
It is difficult to annotate novel SVs or to compute summary data without a reference record or exemplar when multiple SSV choices are available in the same genomic region, and there has been no publicly available resource to date that combines variants from all studies for integration into a bioinformatic pipeline for search, analysis, and comparison. We created structural variant clusters (SVC) to overcome these problems. Structural variant clusters (Figure 1) are smaller discrete genomic features that include counts of the features shared between SSVs. In regions with fuzziness between overlapping SSVs, SCVs allow the calculation of annotation and frequency by either consensus overlapping regions or by userdefined limits.

Additional benefits of having a defined set of SVCs include:

- $\quad$ improved data exchange, data mining, computation, and reporting;

- better searching and matching of genomic coordinates across studies;

- easier aggregation of annotations such as disease and phenotype, frequency, and genomic features that co-locate with a SVC;

- a simplified display in the Sequence Viewer as an aggregated histogram or density track from all studies (currently dbVar display each study as a track, which

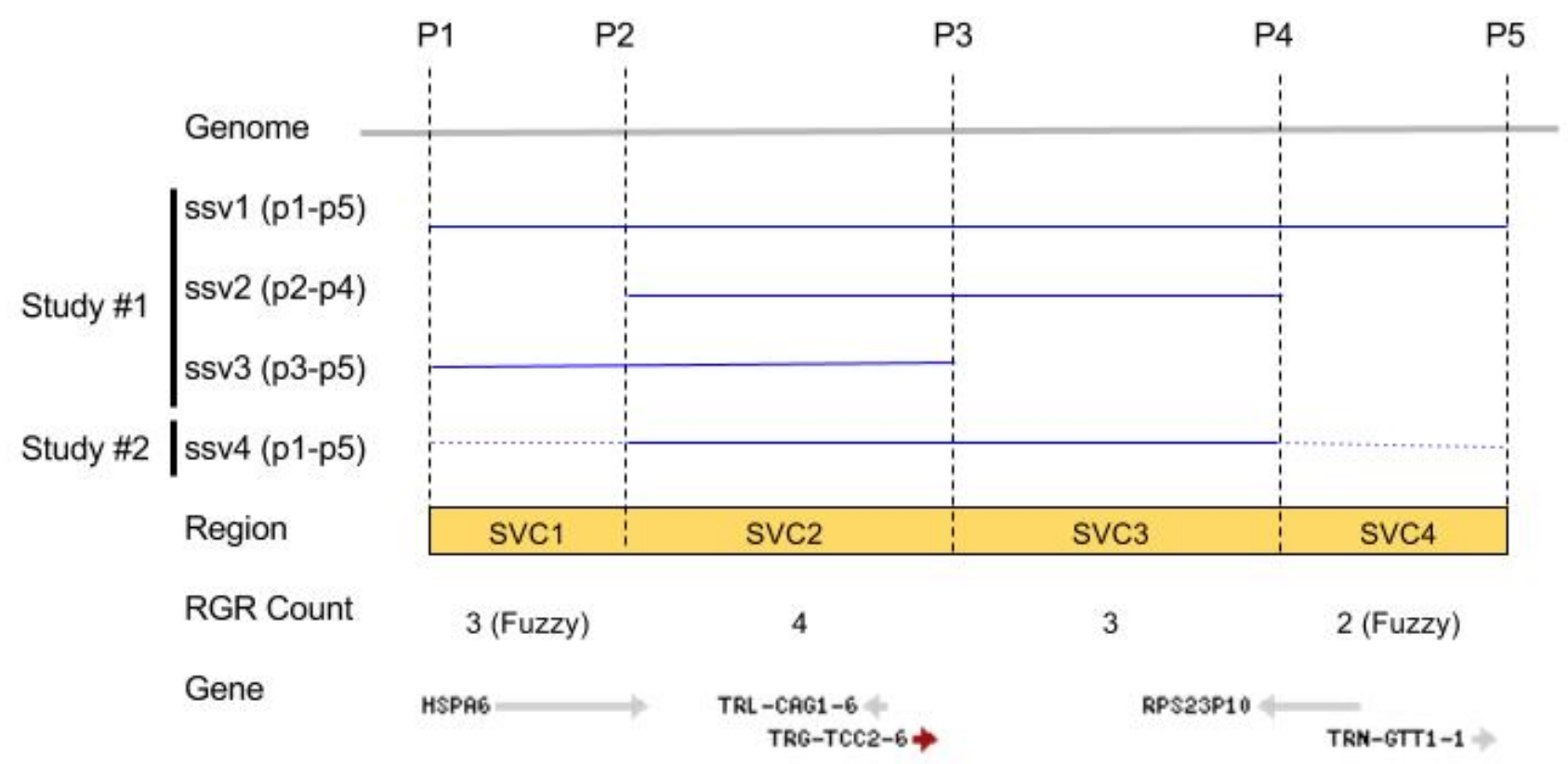

Figure 1. An alignment of variants ssv1-ssv3 (blue lines) with the genome (grey line) between positions P1 and P5. Reference genomic regions SVC1-SVC4 (yellow box) are demarcated by overlap and non-overlapping positions (P1-P2, P2-P3, etc.) between SSVs. The observed SVC counts and the genes are shown on the bottom. 
can be slow to render and difficult to display on small screens); and

- the ability to measure SSV concordance regions and validate across studies.

The Structural Variation Cluster project aimed to accomplish a number of goals. First, we generated a Genome Variant Format (GVF) file of SVC regions as defined above, based on RefSeq GRCh38. Each region is assigned a unique ID (SVC1, SVC2, etc.). The SVC VCF file is used as the basis for generating aggregated data, filtering, generating sequence viewer tracks, and for comparison with user data. We also generated a histogram track to show the frequency of the regions across studies in genomic context for the Sequence Viewer. In addition, we annotated SVC regions with Gene, colocated $\mathrm{dbSNP}^{6}$ reference SNPs, ClinVar ${ }^{7}$, and other colocated features. We aimed to create a tool for filtering SVC GVFs by variant type, region size, region count, chromosome, and additional user-defined splitting and filtering parameters. This tool would allow users to compare their data with SVC GVFs and report matching regions of overlap.

\section{Methods}

SVCs are defined as the union set of overlapping and nonoverlapping regions for all SSVs aligned to the genome using HTSeq version $0.6 .0^{8}$, based on the genomic coordinates in
RefSeq human genome assembly GRCh38 (RefSeq accession GCF_000001405.26) ${ }^{1}$ (Figure 1).

\section{Structural variant cluster (SVC) from SSV}

Figure 2 demonstrates the workflow for this analysis. dbVar SSV data by studies were obtained in tab delimited format from the FTPsite (ftp://ftp.ncbi.nlm.nih.gov/pub/dbVar/data/Homo_sapiens/ by_study/) and used as input. The study files were combined and sorted by chromosome positions into a single file using the script merge_data.py. SVC regions, including counts as shown in Figure 1, were generated from the merged file using the script make_gvf_and_bedgraph.py, which output SVC GVF and BED files. Since the approach in Figure 1 is similar to finding consensus regions or overlapping features between aligned reads make gvf_and_bedgraph.py use HTSeq.GenomicInterval class to store SSV chr. start, and stop coordinates as genomic features and the HTSeq.GenomicArrayOfSets class to identify overlapping positions to generate SVC and counts.

Additional tools are available as scripts using SVC GVF as input to compute summary statistics, to search and filter, to generate WIG files for viewing in sequence viewer, and to annotate using external data sources. All scripts and examples are available on GitHub (https://github.com/NCBI-Hackathons/Structural_Variant_ Comparison/). For this study all coordinates reported are based on GRCh38.

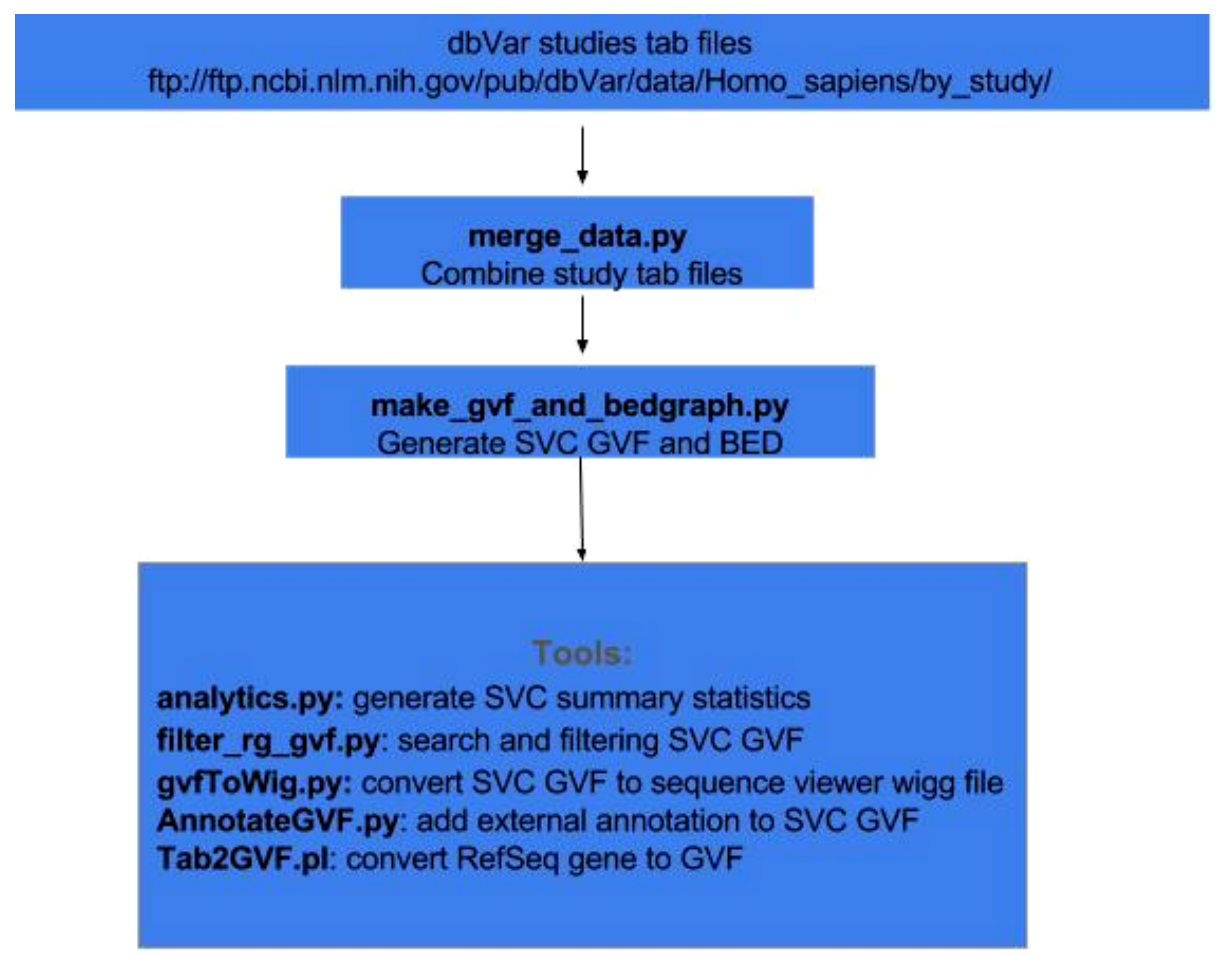

Figure 2. Dataflow for generating SVC and tools for analysis. 


\section{Results}

Computing structural variant cluster (SVC)

As shown in Figure 1, SVCs were created from overlapping and non-overlapping regions of two or more SSVs using the HTSeq.GenomicArrayOfSets class and output as GVF file format. Each SVC is counted for the number of times it is present as a subregion of a SSV, providing a total SVC count across studies. A single SSV by itself without any overlap between itself and another SSV in the region constitutes a single SVC with a feature count of 1. 3.6 million dbVar SSVs generated 3.4 million SVCs for all dbVar data (combined-set) by variant type (Table 1 ).

Comparison of combined-set and study-set SVC derived from variant type CNV with ClinVar and genomic annotations WIG files were generated from SVC GVF files to allow loading into sequence viewer for quick visual inspection as shown in Figure 3. The SVC sets used for inspections are the combinedset which includes 1000 Genomes $^{9}$, as well as other large studies to provide frequently occurring or "common" SVC to compare with presumed curated variants that have clinical significance from study-set (dbVar:nstd37) submitted by ClinGen ${ }^{10}$. The Variation Viewer ${ }^{11}$ allows for quick navigation by genes, chromosome positions, and variations for visual comparison (Figure 3, Figure 4, and Figure 5). Figure 3 and Figure 4 show a hotspot peak A in
Table 1. SVC count and percentage of total by variant types from combined-set across all

studies. The most common variant type was deletion followed by CNV. All CNV types combined (rows 1 , 7 , and 8 in Table 1) total 972,335. We also generated SVC for each variant type (ie. CNV, in/del, etc.) and by individual studies (study-set) for QA/QC and analysis between types and studies of interest. The study-set generated a total of 2.5 million SVCs versus 3.4 million SVCs from the combined-set.

\begin{tabular}{|l|l|l|}
\hline Variant Type & $\begin{array}{l}\text { Percent } \\
\text { Total (\%) }\end{array}$ & SVC Count \\
\hline deletion & $35 \%$ & 1194270 \\
\hline copy number loss & $18 \%$ & 633268 \\
\hline mobile element insertion & $15 \%$ & 517506 \\
\hline duplication & $9 \%$ & 303790 \\
\hline insertion & $7 \%$ & 232552 \\
\hline indel & $5 \%$ & 186658 \\
\hline copy number gain & $5 \%$ & 169876 \\
\hline copy number variation & $5 \%$ & 169191 \\
\hline Others & $1 \%$ & 17810 \\
\hline Total & $100 \%$ & 3424921 \\
\hline
\end{tabular}

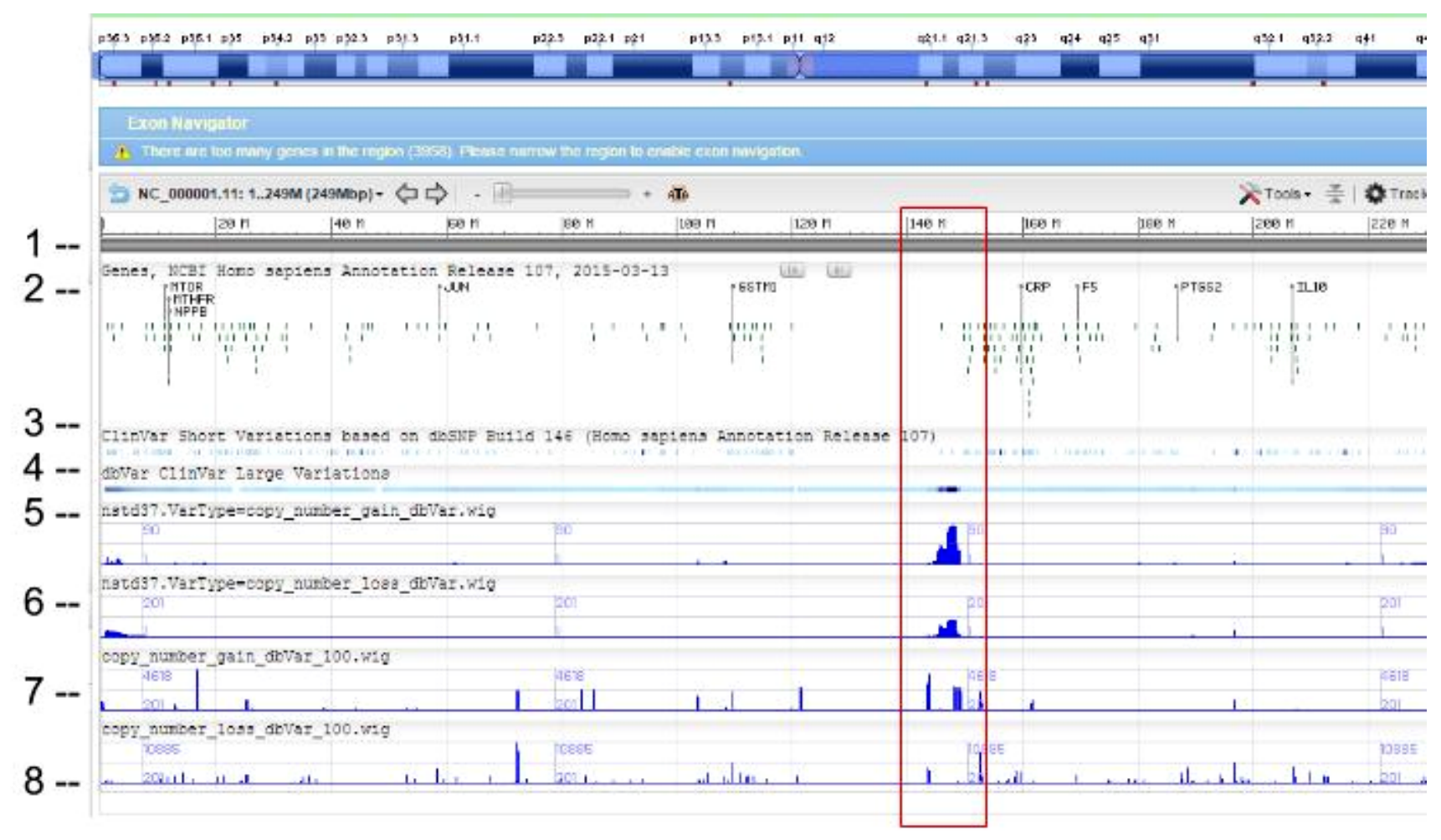

Figure 3. SVC (type=CNV) distribution on chromosome 1 as seen on NCBI Variation Viewer (Variation Viewer - NCBI). Starting from the top: (1) chr 1 sequence, (2) Gene track, (3) ClinVar short variation for dbSNP SNV, (4) ClinVar large variation, (5) ClinGen SVC study-set (dbVar:nstd37) copy number gain, (6) ClinGen SVC study-set (dbVar:nstd37) copy number loss, (7) SVC combined-set for copy number gain with count $>=100$, and (8) SVC combined-set for copy number loss with count $>=100$. The red box highlights an SVC hotspot region found in ClinGen (dbVar:nstd37) tracks 5 and 6 that correspond with the variants in ClinVar. The scale for SVC count histogram are 1-90 (track 5), 1-20 (track 6), 1-4618 (track 7), and 1-10885 (track 8). 


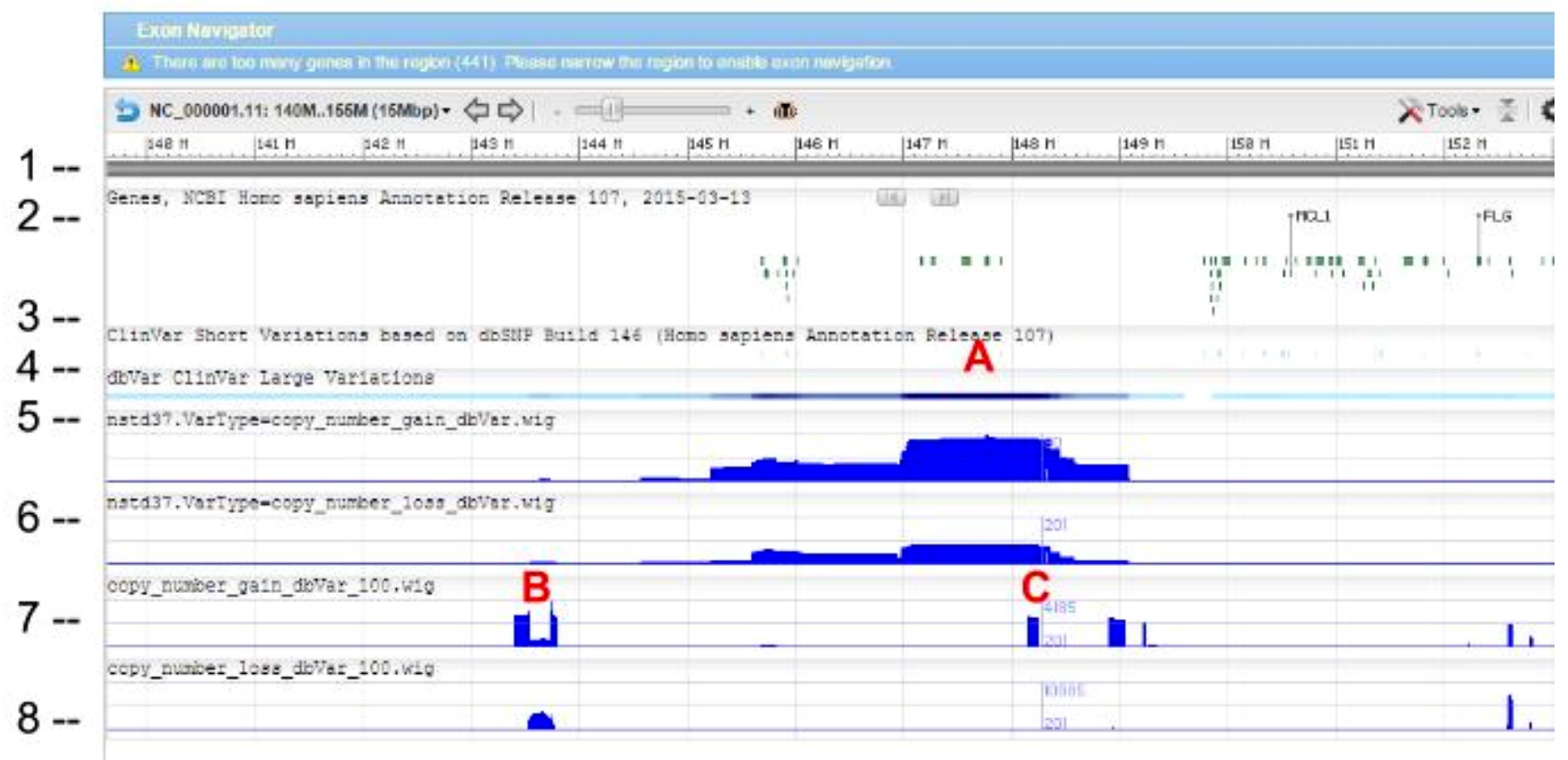

Figure 4. Zoomed in view of the red box region in Figure 1 showing dbVar:nstd37 SVC peak A corresponding to ClinVar variants and flanking peaks $\mathbf{B}$ and $\mathbf{C}$ from the common combined-set. The track and histogram scales are as described in Figure 5.

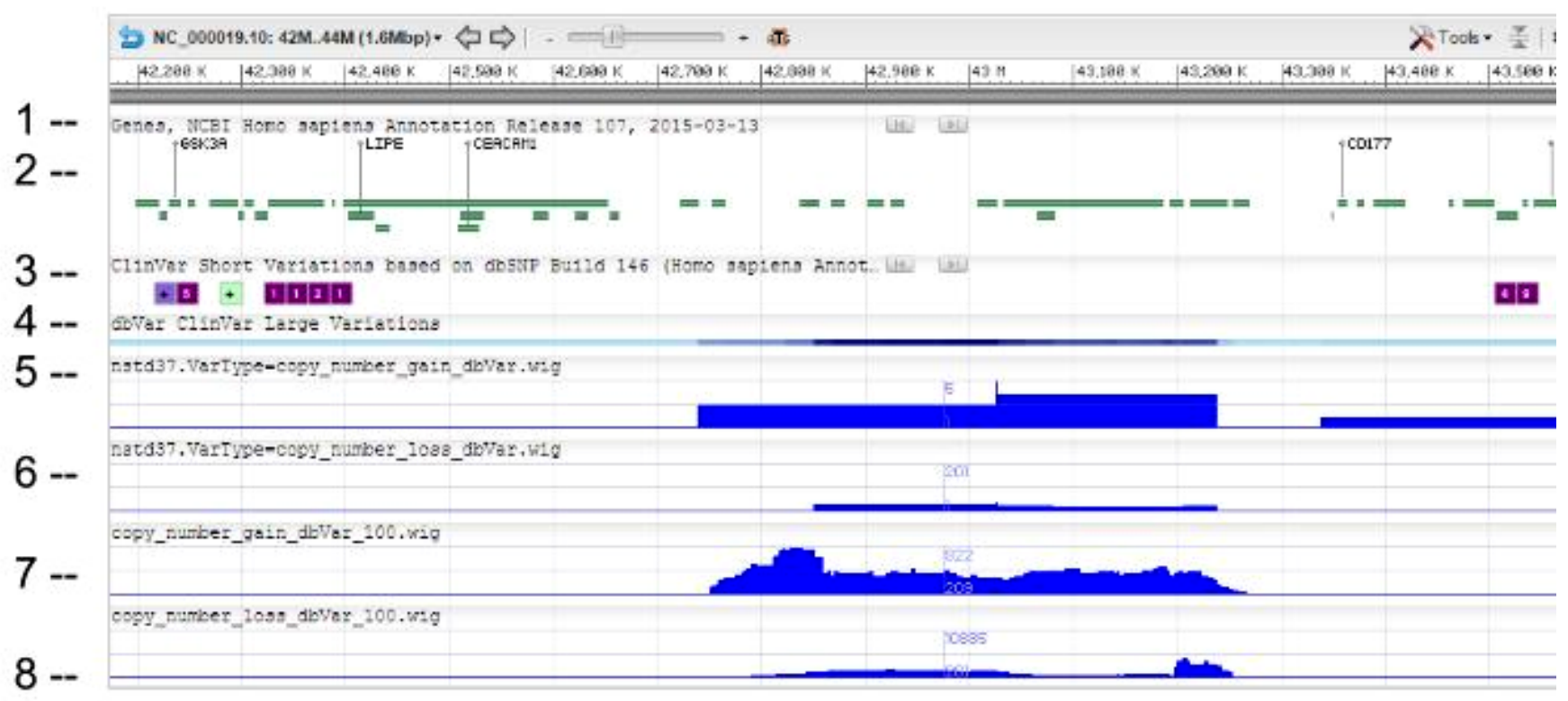

Figure 5. Magnified region of Chr19 showing a region of high density of variants where SVCs presumed to be common are also found. The tracks and histogram scales are as described in Figure 3. 
ClinVar (track 4) that corresponds with a peak in SVC from nstd37, suggesting that this region is critical for function and that variations in this region are rare. These conclusions are supported by the lack of corresponding SVC peaks in the combined-set "common" tracks 7 and 8 . However, tracks 7 and 8 also contain peaks B and C that flank the ClinVar peak, which may demarcate the boundaries for the critical region peak A. In contrast, Figure 5 shows that there are corresponding SVC peaks in the nstd37 (rare) and in the combined-set (common), suggesting that variants in this region may have minimal or no clinical impact by themselves.

\section{Conclusions}

The software tools we developed and provide here compute SVCs and provide counts of concordance regions across SSVs. We also developed tools to search, filter, annotate, and graphically view the results in sequence viewers or to incorporate them into custom analysis pipelines. Using these tools, we provide examples (Figure 3) for comparing across different SVC data sets with other annotation (such as genes and ClinVar). Such comparisons will allow users to investigate across the genome - or near a gene of interest - and to look for concordance and conflicts between data, which may help users form hypotheses regarding the biological impact of observed variation in SVC regions. In future, we will conduct the work and analysis required for SVC data quality assurance. We believe that SVC data promise to improve the analysis and the elucidation of the biological impact of structural variants, and in future, will probably have uses beyond those described here. Potential uses for SVC data could include:

- the evaluation of other SVC hot spot regions to determine if they occur biologically or are due to genome problem regions;

- the use of study metadata to validate SVCs that are in concordance with regions across studies and different assay platforms;

- the validation of rare SVCs $($ count $=<2)$ and common SVCs (count $>2$ );

- identification of evidence of variations in all public SRA data;

- combined analysis and annotation of SVCs to ClinVar, $\mathrm{dbSNP}$, and other variation resources;

- the creation of a reference dbVar "SV" number based on SVCs, which would be the equivalent to dbSNP's RS number;
- identification of population-specific SVCs to gain insight into the functional significance of structural variants and their evolution; and

- determination of high-priority SVCs with significant functional impact and effects.

In addition, a "dbVar Beacon Service" could be developed to allow users to query dbVar if variants exists for a genomic location of interest using combined SVC data. The results would report the number of SVCs and associated SSV IDs and study IDs. Users could then download the study or SSV of interest from dbVar.

\section{Software availability}

Latest source code: https://github.com/NCBI-Hackathons/Structural_Variant_Comparison/

Archived source code as at time of publication: http://dx.doi. org/10.5281/zenodo.48201 ${ }^{12}$

Accompanying wiki: https://github.com/NCBI-Hackathons/Structural_Variant_Comparison/wiki

Manual: https://docs.google.com/document/d/1WBnEnShnw28ZF g17A3xUpWOyvxXjb2q-h1kF-XYVWEw/edit?usp=sharing

License: CC0 1.0 Universal

\section{Author contributions}

All of the authors participated in designing the study, carrying out the research, and preparing the manuscript. All authors were involved in the revision of the draft manuscript and have agreed to the final content.

\section{Competing interests}

No competing interests were disclosed.

Grant information

Lon Phan, John Garner, John Lopez, and Ben Busby's work on this project was supported by the Intramural Research Program of the National Institutes of Health (NIH)/National Library of Medicine (NLM)/NCBI.

The funders had no role in study design, data collection and analysis, decision to publish, or preparation of the manuscript.

Acknowledgements

The authors thank Lisa Federer, NIH Library Writing Center, for manuscript editing assistance. 
1. Saeed S, Bonnefond A, Manzoor J, et al:: Genetic variants in LEP, LEPR, and $M C 4 R$ explain $30 \%$ of severe obesity in children from a consanguineous population. Obesity (Silver Spring). 2015; 23(8): 1687-95.

PubMed Abstract | Publisher Full Text

2. Ross JS, Badve S, Wang K, et al:: Genomic profiling of advanced-stage, metaplastic breast carcinoma by next-generation sequencing reveals frequent, targetable genomic abnormalities and potential new treatment options. Arch Pathol Lab Med. 2015; 139(5): 642-9.

PubMed Abstract | Publisher Full Text

3. Radke DW, Lee C: Adaptive potential of genomic structural variation in human and mammalian evolution. Brief Funct Genomics. 2015; 14(5): 358-68. PubMed Abstract | Publisher Full Text

4. Home - dbVar - NCBI [Internet]: Home - dbVar - NCBI. [cited 2016 Feb 24]. Reference Source

5. Lappalainen I, Lopez J, Skipper L, et al.: DbVar and DGVa: public archives for genomic structural variation. Nucleic Acids Res. 2013; 41(Database issue): D936-41.

PubMed Abstract | Publisher Full Text | Free Full Text

6. Sherry ST, Ward MH, Kholodov M, et al.: dbSNP: the NCBI database of genetic variation. Nucleic Acids Res. 2001; 29(1): 308-11. PubMed Abstract | Publisher Full Text | Free Full Text

7. Landrum MJ, Lee JM, Benson M, et al:: ClinVar: public archive of interpretations of clinically relevant variants. Nucleic Acids Res. 2016; 44(D1): D862-8.

PubMed Abstract | Publisher Full Text | Free Full Text

8. Anders S, Pyl PT, Huber W: HTSeq--a Python framework to work with highthroughput sequencing data. Bioinformatics. 2015; 31(2): 166-9.

PubMed Abstract | Publisher Full Text | Free Full Text

9. estd214 - 1000 Genomes Consortium Phase 3 - dbVar Study - NCBI [Internet]: estd214 1000 Genomes Consortium Phase 3 - dbVar Study - NCBI. [cited 2016 Feb 24] Reference Source

10. ClinGen - ClinGen Clinical Genome Resource [Internet]: ClinGen - ClinGen Clinical Genome Resource. [cited 2016 Feb 24] Reference Source

11. Variation Viewer - NCBI [Internet]: Variation Viewer - NCBI. [cited 2016 Feb 24]. Reference Source

12. John G, TriLe965, Hsu J, et al:: Structural_Variant_Comparison: Initial PostHackathon Release. Zenodo. 2016.

Data Source 


\section{Open Peer Review}

\section{Current Peer Review Status:}

\section{Version 2}

Reviewer Report 06 March 2017

https://doi.org/10.5256/f1000research.10614.r20693

(c) 2017 Zhu L. This is an open access peer review report distributed under the terms of the Creative Commons Attribution License, which permits unrestricted use, distribution, and reproduction in any medium, provided the original work is properly cited.

\section{Lihua Julie Zhu}

Department of Molecular, Cell and Cancer Biology, Program in Bioinformatics and Integrative Biology, University of Massachusetts Medical School, Worcester, MA, USA

Thanks for addressing my comments!

Competing Interests: No competing interests were disclosed.

I confirm that I have read this submission and believe that I have an appropriate level of expertise to confirm that it is of an acceptable scientific standard.

\section{Version 1}

Reviewer Report 09 August 2016

https://doi.org/10.5256/f1000research.8916.r15157

(C) 2016 Zook J. This is an open access peer review report distributed under the terms of the Creative Commons Attribution License, which permits unrestricted use, distribution, and reproduction in any medium, provided the original work is properly cited.

\section{Justin M. Zook}

1 Genome-scale Measurements Group, National Institute of Standards and Technology, Gaithersburg, USA

2 Genome-scale Measurements Group, National Institute of Standards and Technology, Gaithersburg, USA

The authors create a set of scripts that take SVs submitted to dbVar and find how many calls cover each region of the genome. I expect these will be useful for understanding locations in the 
genome where multiple SV calls have been made. The methods appear to be straightforward to use, so that they can be applied to new callsets as they are submitted to dbVar and potentially to other repositories as well. I have a few minor suggestions below:

1. Fig 4 caption seems to refer to red box in Fig 3, not Fig 1.

2. It appears that the output wig files for the current dbvar are on the GitHub site, and it would be useful to make clear that these are available in the paper. Are the output bed files also available? Are the outputs available as a track in any NCBI browser?

3. Why did the authors choose gvf as the output format? Although no format is great for SVs, would the authors consider adding vcf as an output format since vcf seems to be increasingly adopted by SV callers?

4. This is implied in the future work proposed, but it may be useful to state explicitly that dbVar entries are not curated for accuracy, so regions with many SVs may be enriched for artifacts or true SVs or both.

Competing Interests: No competing interests were disclosed.

\title{
I confirm that I have read this submission and believe that I have an appropriate level of expertise to confirm that it is of an acceptable scientific standard.
}

Reviewer Report 05 May 2016

https://doi.org/10.5256/f1000research.8916.r13373

(C) 2016 Zhu L. This is an open access peer review report distributed under the terms of the Creative Commons Attribution License, which permits unrestricted use, distribution, and reproduction in any medium, provided the original work is properly cited.

\author{
Lihua Julie Zhu \\ ${ }^{1}$ Department of Molecular, Cell and Cancer Biology, Program in Bioinformatics and Integrative \\ Biology, University of Massachusetts Medical School, Worcester, MA, USA \\ 2 Department of Molecular, Cell and Cancer Biology, Program in Bioinformatics and Integrative \\ Biology, University of Massachusetts Medical School, Worcester, MA, USA
}

dbVar is a database hosted by NCBI for archiving all types of genomic structural variants (GSV) in all species, including copy number variations (CNV), insertions, deletions, inversions, translocations, and complex chromosomal rearrangement. It accepts data submissions from researchers and exchanges data on a regular basis with the European Database of Genomic Variants Archive (DGVa). To facilitate the exchange, annotation, computation, visualization, reporting and interpretation of user submitted structural variants (SSVs), overlapping SSVs are merged to form a non-redundant set of genomic regions called structural variant clusters (SVCs), and utilities have been developed for annotating, searching, summarizing, filtering and visualizing SVC data in GVF format. However, in light of the previous publication of the same database (Lappalainen et al., 2013), it is unclear to the reviewers about the additional contribution of this 
manuscript. It would be important if the authors could cite the previous publication and clearly describe detailed updates made to the database and how these updates improve the existing software to help reviewers to understand what is new.

It would be helpful if the authors could clarify the reason why Table 1 does not add up to $100 \%$, and describe where 2.5 million SVCs are derived from as stated as "The study-set generated a total of 2.5 million SVCs versus 3.4 million SVCs from the combined-set ". In addition, the resolution for Figure 2-5 needs to be improved.

\section{References}

1. Lappalainen I, Lopez J, Skipper L, Hefferon T, et al.: DbVar and DGVa: public archives for genomic structural variation.Nucleic Acids Res. 2013; 41 (Database issue): D936-41 PubMed Abstract | Publisher Full Text

Competing Interests: No competing interests were disclosed.

I confirm that I have read this submission and believe that I have an appropriate level of expertise to confirm that it is of an acceptable scientific standard, however I have significant reservations, as outlined above.

The benefits of publishing with F1000Research:

- Your article is published within days, with no editorial bias

- You can publish traditional articles, null/negative results, case reports, data notes and more

- The peer review process is transparent and collaborative

- Your article is indexed in PubMed after passing peer review

- Dedicated customer support at every stage

For pre-submission enquiries, contact research@f1000.com 